\title{
Metamemory in schizophrenia: Retrospective confidence ratings interact with neurocognitive deficits
}

\author{
Sarah Eifler ${ }^{\mathrm{a}, *}$, Franziska Rausch ${ }^{\mathrm{a}}$, Frederike Schirmbeck ${ }^{\mathrm{a}, \mathrm{d}}$, Ruth Veckenstedt ${ }^{\mathrm{c}}$, \\ Daniela Mier ${ }^{\mathrm{b}}$, Christine Esslinger ${ }^{\mathrm{e}}$, Susanne Englisch ${ }^{\mathrm{a}}$, Andreas Meyer-Lindenberg ${ }^{\mathrm{a}}$, \\ Peter Kirsch ${ }^{\mathrm{b}}$, Mathias Zink ${ }^{\mathrm{a}}$ \\ ${ }^{a}$ Department of Psychiatry and Psychotherapy, Central Institute of Mental Health, University of Heidelberg/Medical Faculty Mannheim, J5, \\ 68159 Mannheim, Germany \\ ${ }^{\mathrm{b}}$ Department of Clinical Psychology, Central Institute of Mental Health, University of Heidelberg/Medical Faculty Mannheim, J5, 68159 Mannheim, Germany \\ ${ }^{c}$ Department of Psychiatry and Psychotherapy, University Medical Center Hamburg-Eppendorf, 20246 Hamburg, Germany \\ ${ }^{d}$ Department of Psychiatry, Academic Medical Center, University of Amsterdam, Meibergdreef 5, 1105 AZ Amsterdam, The Netherlands \\ ${ }^{\text {e }}$ AWO Health Center, Dr--Grosz-Straße 1-2, 39126 Magdeburg, Germany
}

Keywords:

Cognition

Cognitive biases

Delusion

Memory confidence

Metacognition

Neuropsychiatry

\begin{abstract}
A B S T R A C T
Prior studies with schizophrenia patients described a reduced ability to discriminate between correct and false memories in terms of confidence compared to control groups. This metamemory bias has been associated with the emergence and maintenance of delusions. The relation to neuropsychological performance and other clinical dimensions is incompletely understood. In a cross-sectional study, metamemory functioning was explored in 32 schizophrenia patients and 25 healthy controls. Metamemory was assessed using a verbal recognition task combined with retrospective confidence level ratings. Associations of metamemory performance with six neuropsychological domains (executive functioning/problem solving, speed of processing, working memory, verbal and visual learning, and attention/vigilance) and psychopathological measures were analyzed. Results revealed a significantly smaller discrepancy between confidence ratings for correct and incorrect recognitions in the patient group. Furthermore, patients showed significantly lower recognition accuracy in the metamemory task and marked deficits in all neuropsychological domains. Across all participants, metamemory performance significantly correlated with executive functioning and working memory. No associations with delusions were found. This data confirms prior findings of metamemory biases in schizophrenia. Selective neuropsychological abilities seem to be modulating factors of metamemory functioning. Longitudinal studies in at risk mental state and first-episode patients are needed to reveal causal interrelations.
\end{abstract}

\section{Introduction}

In general, memory abilities are among the most severely impaired cognitive functions in schizophrenia (Aleman et al., 1999; Heinrichs and Zakzanis, 1998; Fioravanti et al., 2005). Several aspects of memory are affected (Gras-Vincendon et al., 1994; Lee and Park, 2005; Assaf et al., 2007; Leavitt and Goldberg, 2009). But in addition to the analysis of memory accuracy, more qualitative examinations of memory processes have recently concentrated on reflection- and monitoring processes of one's

\footnotetext{
* Correspondence to: Central Institute of Mental Health, Department of Psychiatry and Psychotherapy Medical Faculty Mannheim / Heidelberg University P.O. Box 122120 D-68072 Mannheim, Germany. Tel.: +49621 1703 2523; fax: +49621 17031205 .

E-mail address: sarah.eifler@zi-mannheim.de (S. Eifler).
}

own memory, so-called metamemory processes (Flavell, 1971). A good strategy to gain insight into various aspects of these monitoring abilities is to explore peoples' subjective judgments of their memory performance (Shimamura, 2000). One way to explore knowledge about memory abilities is to use self-rating scales. For example, Bacon et al. (2011) found that schizophrenia patients reported lower memory capacity, marginally lower mastery over their memory functions as well as less access to memory strategies compared to healthy controls. Another way is to ask for patients' introspective judgments of their actual performance in memory tasks. This can be done at different temporal stages of the memory process (Nelson and Narens, 1994). For example, judgments-of-learning can be given at the time of encoding, regarding upcoming memory retrieval. Second, feeling-ofknowing statements can be given about the likelihood that a correct answer will be recognized in future, after some 
information has not been successfully retrieved. Third, confidence level ratings about a given answer can be indicated retrospectively at the time of retrieval. Using these methods, impaired metamemory abilities of schizophrenia patients were found compared to healthy and psychiatric control participants (Bacon et al., 2001, 2007; Kircher et al., 2007; Moritz et al., 2006d, 2006e, 2008).

Retrospective confidence level ratings, measuring cognitive confidence, have been shown to be valid indicators of the subjective accuracy of memory retrieval (Moritz et al., 2011). Prior studies found support for a biased evaluation of memory performance in schizophrenia patients using these confidence level ratings (Danion et al., 2001; Moritz and Woodward, 2006b; Moritz et al., 2003, 2005; Peters et al., 2013). More recently, similar results have also been detected in studies regarding source monitoring (Gaweda et al., 2012, 2013) and social judgments (Köther et al., 2012; Moritz et al., 2012). A main bias seems to be an increased confidence in incorrect memories in addition to a decreased confidence in correct responses compared to healthy people and psychiatric controls (Moritz and Woodward, 2006b; Moritz et al., 2008; Peters et al., 2013). This phenomenon has been described as a decreased "confidence gap", i.e. a discrepancy between the certainty for correct and incorrect responses. Another bias of metamemory ability has been termed "knowledge corruption" (Moritz et al., 2006d). The research group found schizophrenia patients to have an increased proportion of errors which they judged with very high confidence to be correct. The knowledge corruption index thus describes high-confident subjective, but false knowledge (Moritz et al., 2008).

Overconfidence in memory errors may represent a risk factor for delusions and hallucinations (Moritz and Woodward, 2006a; Gaweda et al., 2013). It might be a consequence of a liability to accept false hypotheses as true and evaluate them on the basis of too little information-a liberal acceptance-which in turn results in wrong and delusional interpretations of situations (Moritz et al., 2005, 2008), comparable to the "jumping to conclusion" bias (Fine et al., 2007). Some investigations suggested this bias to be schizophrenia-specific (Moritz and Woodward, 2004, 2006a, 2006b). Correlations between overconfidence in memory and delusions were not only found in schizophrenia patients (Moritz and Woodward, 2002) but also in healthy controls with schizotypic properties (Corlett et al., 2009). However, findings of overconfidence and knowledge corruption in patients with alcohol dependence and a history of hallucinations rather point to more general associations with positive symptoms in different illnesses (Gaweda et al., 2014). There are also studies with schizophrenia patients which did not reveal any association (Kircher et al., 2007; Peters et al., 2013).

So far, the literature lacks comprehensive examinations of associations between metamemory function and neuropsychological performance. The existing studies reported inconsistent results and only a small number of studies included behavioral designs to test metamemory abilities. In healthy controls, correlations between items of metacognitive rating scales and executive functions (updating, shifting and inhibition) were described (Mäntylä et al., 2010). Furthermore, divided attention at encoding in a verbal memory task resulted in overconfidence in memory prediction in healthy controls (Sacher et al., 2009). In schizophrenia patients, some studies reported correlations between metacognitive rating scales and cognitive performance, as executive functions (Bacon et al., 2011; Lysaker et al., 2008; Souchay et al., 2004), whereas others discussed independence between metamemory deficits and executive function as well as verbal memory (Moritz et al., 2003), attention and immediate memory (Kircher et al., 2007) and intelligence measures (Kircher et al., 2007; Moritz et al., 2003, 2005). To shed light on associations between metamemory and neurocognitive abilities and to understand possible modulating effects, more detailed investigations seem necessary.

This study aimed at replicating findings regarding metamemory abilities in a well characterized sample of schizophrenia inpatients compared to healthy control participants. First, in order to assess retrospective confidence level ratings of memory, we used a variant of the Deese-Roediger-McDermott (DRM) paradigm (Deese, 1959), a verbal false memory task. Second, we aimed at evaluating correlations between psychosis symptoms and cognitive confidence in memory. Third, we intended to address the basic cognitive mechanisms of retrospective cognitive confidence in memory using the comprehensive neuropsychological battery Measurement and Treatment Research to Improve Cognition in Schizophrenia (MATRICS) Consensus Cognitive Battery (MCCB; Kern et al., 2011; Nuechterlein et al., 2008) to extend the literature regarding mutual interrelations between neurocognition and metacognitive biases in schizophrenia. Referring to the previous literature, we hypothesized that patients with schizophrenia show a decreased confidence gap and an increased knowledge corruption index compared to healthy participants, indicating metamemory biases. In secondary analyses, we addressed the following research questions: Is confidence in errors associated with positive symptoms in schizophrenia? Are there associations between the performance in neurocognitive domains and overconfidence in errors?

\section{Methods}

\subsection{Study design and participants}

The present study was designed as a cross-sectional parallel-group comparison and was part of a comprehensive investigation of metacognition in schizophrenia. It was approved by the ethical board of the Ruprecht-Karls-University Heidelberg (accession number: 2009-296 N-MA). Analyses of endpoints beyond metamemory included partially overlapping participants (Eifler et al., 2014; Rausch et al., 2014). All participants were informed about aims and procedures of the study and provided their written consent after a sufficient period of consideration and resolving open questions. A number of 32 patients with schizophrenia were recruited during their inpatient treatment at the Central Institute of Mental Health $(\mathrm{CIMH})$ in Mannheim, Germany. Seven patients fulfilled symptomatic remission according to Andreasen et al. (2005) with PANSS scores $\leq 3$ on remission criteria items. All of them fulfilled a set of predefined inclusion criteria: diagnosis of schizophrenia according to the Diagnostic and Statistical Manual (DSM-IV-R; Saß et al., 2000), age between 18 and 60 years, ability to provide informed consent, sufficient German language skills and residence within a $50 \mathrm{~km}$ radius around Mannheim. We did not include patients with substance dependence (excluding nicotine), other disorders of the central nervous system requiring treatment and patients receiving combined antipsychotic treatment or augmentation with antidepressants or mood stabilizers. Current antipsychotic treatment with second generation antipsychotic agents was quantified using chlorpromazine (CPZ) equivalents (Andreasen et al., 2010). Twelve patients were treated with benzodiazepines (lorazepam: $n=12$, mean dosage: 2.0 (S.D.: 1.57 ) $\mathrm{mg} /$ day) because of anxiety, agitation, or sleep disorder. No patient was treated with anticholinergic agents. 25 control subjects were matched on group level according to gender, age and levels of education. They were carefully characterized regarding family and previous medical history before study entry. Subjects with suicide, schizophrenia and bipolar disorder in first-degree relatives, as well as subjects with disorders of the central nervous system, inpatient treatment in psychiatric hospitals, current treatment with psychotropic agents, substance dependence (excluding nicotine) and abuse of illegal substances within the 4 weeks before investigation were excluded. In addition, a screening regarding currently present psychopathological syndromes according to DSM-IV-R was performed using the M.I.N.I. (Mini-International Neuropsychiatric Interview; Sheehan et al., 1998).

\subsection{Psychopathology}

Current psychopathology was assessed by trained raters (either by S.E. or F.R). Training was established by standardized video-based sessions. No psychopathological scales were rated by both raters for the same patient and no inter-rater reliability data was collected. The Positive and Negative Syndrome Scale (PANSS; Kay et al., 1987) was used to assess positive and negative symptoms as well as the general psychopathology. Besides these scale-sumscores, we were interested in single PANSS items representing delusions (P1) and hallucinations (P3). Furthermore, the delusion part of the Psychotic Symptom Rating Scales (PSYRATS; Haddock et al., 1999) was used to measure qualitative aspects of delusions in six domains (preoccupation with delusions, duration of preoccupation, delusional conviction, amount of distress, intensity of distress and disruption of life). The PSYRATS is a semi-structured interview. Every item can be rated on a five-point scale ranging from 0 to 4 . A 
composite score is expressed by the sum of all items. Each level is explained by an anchor point which facilitates a direct translation of the patient's answer into numbered ratings. Good inter-rater reliability, test-retest reliability, internal consistency and validity have been reported (Haddock et al., 1999; Drake et al., 2007). The authorized German version of the PSYRATS was translated and back-translated with satisfactory psychometric properties (Schneider et al., 2011). The Scale for the Assessment of Negative Symptoms (SANS; Andreasen, 1989) and the Calgary Depression Scale for Schizophrenia (CDSS; Addington et al., 1993) were, in addition to the PANSS, applied to assess negative and depressive symptoms. Social and global functioning were rated using the scale for the General Assessment of Functioning (GAF; Jones et al., 1995) and the Personal and Social Performance Scale (PSP; Patrick et al., 2009). Severity of illness was assessed with the Clinical Global Impression Scale (CGI; National Institute of Mental Health [NIMH], 1970).

\subsection{Metamemory task}

Metamemory performance was assessed using a computerized variant of the Deese-Roediger-McDermott paradigm (Deese, 1959; Roediger and McDermott, 1995), programmed on Presentation Version 14.4 (Neurobehavioral Systems Inc, Albany, CA). Stimuli for the paradigm had been validated prior to our study with 55 healthy participants. A detailed description of the stimuli generation and standardization processes can be found in a prior publication (Moritz et al., 2006c). Participants were asked to spontaneously give 10 associations for a set of 114 theme words. Subsequently, lists were compiled which included 16 association words (including the theme word) in descending order with respect to their response frequency. In our study, we extracted six of the generated wordlists which were associated with the following themes: holiday, street, betrayal, love, to look and garbage. One word with high frequency (drawn from the five words with highest associative strength regarding the theme word), one word with medium frequency (drawn from the words on location $6-10$ on the association list), one word with low frequency (drawn from the five words with lowest associative strength) as well as the theme word itself were extracted from the 16 words generated per lists to serve as lures in the recognition phase.

The task design was blocked (encoding-recognition-encoding-recognition). Three wordlists were provided per block. Encoding blocks started with following instructions: "You will see different words. Try to memorize these words. Afterwards, you will be asked if you have seen the words before". Instructions were variable in duration and ended by a key-press of the participant. During encoding, the remaining 12 words per list were presented sequentially one second each with intermediate intervals of two seconds. They were presented in descending semantic association with the theme word. A 10-second-interval was included before the presentation of a new word list. Each encoding phase thus lasted 2:18 min in total. Lists were presented in the above depicted order (starting with the association list of holiday). The recognition block was introduced by instructions of variable duration, which disappeared after a key-press. Instructions were read as follows: "You will now see different words. Try to remember if you have seen the word before. Please indicate how certain you are about your decision". During the recognition phase, 12 words per list were presented. The recognition list contained six words that had actually been presented, two new words that were not related to the theme word, and another four words that had not been presented but were semantically related to the studied words (the lure words, including the theme word as critical lure). Confidence was rated on a Likert scale. Patients had to indicate if they were $100 \%$ certain, rather certain or uncertain about seeing a word or not, respectively. Each word remained on the screen until the participant made a decision by a key-press.

Variables of interest were the amount of hits (correctly recognized studied words), correct lure rejections (correct rejections of new words which were semantically related), misses (not recognized but studied words) and false positive lures (new words, semantically related and incorrectly judged as studied). Consistent with work from Moritz and Woodward (2006b), we computed two metamemory parameters. First, the confidence gap was calculated by subtracting the mean confidence in incorrect answers from the mean confidence in correct answers. This index includes all confidences in any type of answer. The confidence gap is an index of the participants' ability to discriminate false and true memories in terms of confidence. Second, the knowledge corruption index was calculated by generating the percentage of very high-confident answers (only answers with 100\% certainty) which are errors (sum of high-confident errors/sum of total highconfident responses (correct+errors) $\times 100$ ). The knowledge corruption index specifically relates to subjective, but false, knowledge.

\subsection{Neurocognitive abilities}

Twelve tests were applied in order to assess six neuropsychological domains executive functioning/problem solving, speed of processing, working memory, verbal and visual learning and attention/vigilance. These domains are recommended for cognitive assessment in schizophrenia by the MATRICS initiative and combined in the MCCB (Kern et al., 2011; Nuechterlein and Green, 2006; Nuechterlein et al., 2008). In detail, executive functioning was evaluated with the Wisconsin Card Sorting Test (WCST; Drühe-Wienholt and Wienholt, 1998) and a mazes-task (Iverson et al., 2008), speed of processing with the trail making tests part A and B (TMT-A/B; Reitan and Wolfson, 1995), a symbol coding task (Brief
Assessment of Cognition in Schizophrenia (BACS)-symbol coding; Keefe et al., 2008) and a verbal fluency task (animal naming), working memory with the letternumber-sequencing (LNS) and a block tapping task (spatial span) of the Wechsler Memory Scale (Wechsler, 1997). Furthermore, we assessed verbal learning with the Hopkins' verbal learning task revised (HVLT-R; Benedict et al., 1998), visual learning with the brief visuo-spatial memory test revised (BVMT-R; Benedict, 1997) and vigilance with the continuous performance test, subtest identical pairs (CPT-IP; Knye et al., 2004). All tests were standardized by the MATRICS initiative and test scores as well as domain scores, expressed by $t$-values, were calculated with the help of a computerized analysis program (Nuechterlein and Green, 2006). All tests measuring one domain contribute equally to the domain score. In addition, premorbid verbal intelligence was measured using the Multiple Choice Word Test (MWT, version B; Lehrl, 2005). MWT-B data of three schizophrenia patients did not represent their actual verbal intelligence because patients did not have a native German background. Therefore their data was excluded from all between-group analyses and correlational analyses which included neurocognitive variables.

\subsection{Statistics}

Sociodemographic, psychometric and behavioral parameters were analyzed using the Statistical Package for Social Sciences (SPSS version 18.0, Chicago, IL, US). Sample-size estimations were done using the statistical software $G^{*}$ power 3.1 (Faul et al., 2007). According to prior data evaluating the confidence gap in schizophrenia patients (Moritz et al., 2006d; Moritz et al., 2008), considering a power of 0.8 , $\alpha=0.05$ and a more conservative effect size (Cohen's $d=0.8$ ), a sufficient sample size of at least 26 was calculated. We tested for group differences by applying student's $t$-tests, analyses of covariance, as well as Chi-square-tests. Group affiliation served as between-subjects variable, cognitive outcomes as dependent variables. When assumptions were not met, non-parametric Mann-Whitney- $U$ Tests were applied. Correlation analyses were performed using standard nonparametric Spearman's rho coefficient regarding associations between metamemory and positive symptoms and Pearson product-moment correlations or standard non-parametric Spearman's rho coefficient for neuropsychological performance (total sample). Bonferroni corrections for multiple testing were used to safeguard against an inflation of Type I error (metamemory: $\alpha=0.05 / 2=0.025$, neuropsychology: $\alpha=0.05 / 10=0.005$, correlations between psychopathology and metamemory: $\alpha=0.05 / 18=0.003$, correlations between neuropsychology and metamemory: $\alpha=0.05 / 16=0.003$ ).

\section{Results}

\subsection{Sociodemographic, clinical and neuropsychological characteristics}

Groups did not differ regarding age, gender and years of education. The estimated premorbid verbal intelligence differed between groups, but did not have a significant influence on neuropsychological $(p=0.08)$ or metamemory $(p=0.77)$ performance. Therefore, subsequent analyses were performed using analyses of variance. A detailed overview regarding sociodemographic characteristics is provided in Table 1.

Schizophrenia patients showed mild to moderate psychotic symptoms according to a mean PANSS total score of 67.41 (S.D. $=16.01$ ), PANSS item P1 of 2.81 (S.D. $=1.53$ ), PANSS item P3 of 2.28 (S.D. $=1.35$ ) and further represented in the PSYRATS ratings (Table 2). SANS and CDSS scores indicated moderate negative and rather low depressive symptoms. Moderate severity of illness was suggested by the CGI, but marked psychosocial- and general dysfunctions by GAF and PSP.

Regarding neuropsychology, schizophrenia patients performed significantly lower in all domains, indicating impairment in the domains executive functioning, attention, speed of processing, working memory, verbal and visual learning, problem solving and vigilance (see Supplementary Table 1).

\subsection{Metamemory task}

Overall, wordlists did not differ regarding associative strength $(p=0.49)$ and word length $(p=0.89)$ of the presented items. Furthermore, lure words and studied words did not differ in their 
Table 1

Sociodemographic data and clinical characteristics.

\begin{tabular}{|c|c|c|c|c|}
\hline & \multirow{2}{*}{$\begin{array}{l}\text { Patients } \\
(n=32)\end{array}$} & \multirow{2}{*}{$\begin{array}{l}\text { Controls } \\
(n=25)\end{array}$} & \multicolumn{2}{|c|}{ Group comparison } \\
\hline & & & Test & $p$ \\
\hline \multicolumn{5}{|l|}{ Sociodemographics } \\
\hline Age & $37.22(9.68)$ & $38.12(10.72)$ & $t(55)=0.33$ & 0.70 \\
\hline Sex (male/female) & $24 / 8$ & $19 / 6$ & $\chi^{2}(1)=0.008$ & 0.90 \\
\hline Years of education & $14.41(2.79)$ & $15.24(2.83)$ & $t(55)=1.11$ & 0.20 \\
\hline Premorbid intelligence (MWT-B) & IQ: $98.45(13.89)(n=29)$ & IQ: $107.6(13.82)$ & $U=208.5$ & $0.007^{*}$ \\
\hline \multicolumn{5}{|l|}{ Clinical properties } \\
\hline Years of illness & $8.75(6.72)$ & - & - & - \\
\hline Previous hospitalizations & $5.0(6.66)$ & - & - & - \\
\hline Chlorpromazine equivalent doses(mg) & $425.96(183.68)$ & - & - & - \\
\hline Duration of medication (days) & $53.16(74.16)$ & & & \\
\hline
\end{tabular}

Note. Data is illustrated in means (standard deviations). $\mathrm{IQ}=$ intelligence quotient; MWT-B=Multiple Choice Word Test, version $\mathrm{B}$.

$* p<0.01$.

Table 2

Psychopathological data of the patient sample.

\begin{tabular}{lc}
\hline & Patients $(n=32)$ \\
\hline PANSS & \\
Total score & $67.41(16.01)$ \\
Positive symptoms & $15.75(5.34)$ \\
Negative symptoms & $16.59((6.54)$ \\
General psychopathology & $35.06(8.7)$ \\
PSYRATS & \\
Total score & $6.93(7.12)$ \\
Preoccupation & $1.31(1.26)$ \\
Conviction & $1.34(1.52)$ \\
Distress & $1.13(1.39)$ \\
Intensity of distress & $1.44(1.54)$ \\
Disruption & $1.13(1.19)$ \\
SANS & $25.34(18.87)$ \\
CDSS & $3.38(3.46)$ \\
PSP & $48.84(16.05)$ \\
GAF & $42.84(10.7)$ \\
CGI-S & $4.44(0.95)$ \\
\hline
\end{tabular}

Note. Data is illustrated in means (standard deviations). $\mathrm{CDSS}=\mathrm{Cal}-$ gary Depression Scale for Schizophrenia; CGI-S=Clinical Global Impression Scale- Severity of Illness; $\mathrm{GAF}=$ General Assessment of Functioning; PANSS $=$ Positive and Negative Syndrome Scale: $\mathrm{PSP}=$ Personal and Social Performance Scale; PSYRATS = Psychotic Symptom Rating Scale, delusion scale; SANS=Scale for the Assessment of Negative Symptoms.

associative strength to the theme word $(p=0.58)$ or in word length $(p=0.89)$.

Regarding memory accuracy, groups significantly differed in the number of correctly recognized studied words (hits) and in the number of misses after Bonferroni correction (Table 3).

Regarding confidence level ratings, group comparisons revealed a significant lower confidence gap in the patient group after Bonferroni correction with a medium-large effect. The knowledge corruption index showed a trend towards a group difference. The effect sizes indicated a medium-large effect. Metamemory data is summarized in Table 3. The confidence gap did not correlate with the total number of errors (misses and false positives) in the metamemory task (patients: $\rho=-0.147, p=0.42$; controls: $\rho=0.08, p=0.70$ ).

\subsection{Correlational analyses}

Correlational analyses revealed no significant correlations between metamemory and the severity of psychotic symptoms, in particular delusions, as revealed by PANSS or PSYRATS (Table 4). Furthermore, the confidence gap did not correlate with the dosage of benzodiazepines $(\rho=-0.08, p=0.67)$ nor the CPZ equivalent dose $(\rho=0.05, p=0.81)$. Groups with and without lorazepam did not differ significantly in the confidence gap $(t(27)=1.08, p=0.29)$.

In an explorative analysis including Bonferroni-correction, we detected significant correlations between the knowledge corruption index and executive functioning, represented in WCST outcome measures "\% errors" and "\% perseverative errors", and the MCCB domain working memory. Furthermore, significant associations were found between the confidence gap and WCST “\% errors" (Table 4).

\section{Discussion}

The aims of this study were to evaluate metamemory performance in schizophrenia patients and to explore the interplay between metamemory functioning and dimensions of psychopathology and neuropsychological performance. In line with our hypothesis, our results confirm prior findings of a biased evaluation of memory performance in schizophrenia patients. Metamemory biases were not specifically associated with positive symptoms. However, metamemory performance was associated with several neurocognitive abilities.

Replicating earlier findings, schizophrenia patients had significant lower recognition accuracy for studied words. Furthermore, we found a reduced confidence gap in the patient group (compare Moritz and Woodward, 2006a, 2006b; Moritz et al., 2008) indicating a biased ability to differentiate between correct and incorrect memories in terms of confidence. These results suggest that schizophrenia patients show two levels of memory impairment: first, an accuracy deficit and second, a deficit in monitoring this memory failure sufficiently in terms of differentiating between correct and false. As a consequence, these cognitive biases may trigger decisions on the basis of erroneous assumptions. The knowledge corruption index was not, as hypothesized, significantly higher in the patient group. This is an unexpected finding because many other studies have found an increased knowledge corruption index (e.g. Moritz et al., 2004, 2008) in similar patient groups. A probable reason is that our conservative methods, using Bonferroni correction, contributed to this result. This method was used to control for multiple testing of these interrelated constructs. Important to note, the group difference still reached medium effect size. The non-significant result of the difference in knowledge corruption between schizophrenia patients and healthy people may underestimate the real difference, which should be verified in future studies. Though we conservatively calculated sample sizes beforehand using prior data and included 
Table 3

Group comparison of metamemory performance.

\begin{tabular}{|c|c|c|c|c|c|}
\hline & \multirow{2}{*}{$\begin{array}{l}\text { Patients } \\
(n=29)\end{array}$} & \multirow{2}{*}{$\begin{array}{l}\text { Controls } \\
(n=25)\end{array}$} & \multicolumn{3}{|c|}{ Group comparison } \\
\hline & & & $F$ & $p$ & $d$ \\
\hline \multicolumn{6}{|l|}{ Metamemory accuracy } \\
\hline Hits & $27.66(4.57)$ & $32.12(2.88)$ & $F(1,52)=17.77$ & $0.001^{\mathrm{a}}$ & 0.12 \\
\hline Misses & $8.34(4.57)$ & $3.88(2.88)$ & $F(1,52)=17.77$ & $0.001^{\mathrm{a}}$ & 0.12 \\
\hline Correct lure rejection & $15.10(4.71)$ & $13.76(3.37)$ & $F(1,52)=1.41$ & 0.24 & 0.03 \\
\hline False positive lures & $8.90(4.71)$ & $10.24(3.37)$ & $F(1,52)=1.41$ & 0.24 & 0.03 \\
\hline \multicolumn{6}{|l|}{ Metamemory confidence } \\
\hline Confidence gap & $0.34(0.26)$ & $0.53(0.32)$ & $F(1,52)=5.67$ & $0.021^{\mathrm{a}}$ & 0.65 \\
\hline Knowledge corruption Index & $18.29(9.30)$ & $13.48(5.20)$ & $F(1,52)=5.26$ & 0.026 & 0.63 \\
\hline
\end{tabular}

Note. Data is illustrated in means (standard deviations). Confidence gap: $\overline{\mathrm{x}}$ (confidences incorrect) $-\overline{\mathrm{x}}$ (confidences correct); knowledge corruption index: $\Sigma$ (high-confident errors $) / \Sigma$ (high-confident correct + high-confident errors) $\times 100$.

Group differences were analyzed using analyses of variance (ANOVA).

a Significant group differences after Bonferroni correction for multiple testing ( $\alpha=0.025$ for metamemory confidence; $\alpha=0.005$ for neuropsychological performance including metamemory accuracy).

Table 4

Correlations between metamemory indexes, psychopathology and neuropsychology.

\begin{tabular}{lcc}
\hline & Knowledge corruption index & Confidence gap \\
\hline Psychopathology & & \\
PANSS & 0.08 & \\
Positive Symptoms & -0.11 & -0.01 \\
P1 & -0.33 & 0.01 \\
P3 & 0.31 & 0.20 \\
Negative Symptoms & 0.36 & -0.25 \\
GPP & & -0.25 \\
PSYRATS & 0.17 & \\
Conviction & -0.13 & -0.14 \\
PSP & -0.31 & 0.05 \\
GAF & -0.27 & 0.30 \\
MCCB & & \\
Speed of processing & -0.35 & 0.23 \\
Attention/Vigilance & $-0.39^{\mathrm{a}}$ & 0.11 \\
Working memory & -0.36 & 0.23 \\
Verbal learning & -0.36 & 0.17 \\
Visual learning & -0.16 & 0.12 \\
Reasoning/Problem solving & 0.07 \\
WCST & & \\
\% errors & $0.60^{\mathrm{a}}$ & $-0.41^{\mathrm{a}}$ \\
\% perseverative errors & $0.45^{\mathrm{a}}$ & -0.34 \\
\hline
\end{tabular}

Note. $\mathrm{GAF}=$ Global Assessment of Functioning; GPP=General Psychopathology; $\mathrm{MCCB}=$ MATRICS Consensus Cognitive Battery; PANSS=Positive and Negative Syndrome Scale; PSP = Personal and Social Performance Scale; PSYRATS = Psychotic Symptom Rating Scale; WCST = Wisconsin Card Sorting Test.

Data was analyzed using Pearson product-moment correlations and nonparametric Spearman Rho correlations when assumptions were not met (WCST, clinical ratings).

a Significant results after Bonferroni correction for multiple testing $(\alpha=0.003)$.

sufficient participants according to these prior results, an influence of power can also not be totally ruled out.

It might be assumed that a failure in monitoring one's memory correctly triggers beliefs which are held with strong conviction though they have been proven to be false. In this line, metamemory biases have been regarded risk factors for the emergence and maintenance of delusions (Moritz et al., 2008) and have been associated with hallucinations (Gaweda et al., 2013). Other reasoning biases, as the jumping to conclusion bias (accepting hypotheses on the basis of a very small amount of information) (Fine et al., 2007; Jolley et al., 2014; Rausch et al., 2014) or biases in source monitoring (Moritz et al., 2005; Woodward et al., 2006) have also been associated with delusion development. These cognitive processes in combination might represent psychological factors underlying positive symptoms of schizophrenia. Different mechanisms might be responsible: a tendency towards altered salience attribution to neutral stimuli (Esslinger et al., 2013; Kapur, 2003; Speechley et al., 2010), a deficit in prediction error signaling (Fletcher and Frith, 2009) or complex dopaminergic dysfunctions (Andreou et al., 2013; Fusar-Poli and Meyer-Lindenberg, 2012a, 2012b). Findings of positive correlations between delusions and overconfidence (regardless of correct or false answers) (Moritz and Woodward, 2002), between schizotypy and confidence in memories (Corlett et al., 2009), as well as discussions about the specificity of metamemory biases to schizophrenia (compare Moritz and Woodward, 2006a), support this assumption. However, the present results and other investigations (e.g. Kircher et al., 2007; Moritz et al., 2003) did not reveal immediate and significant associations between positive symptoms and metamemory biases. It might be suggested that the evaluated indexes of metamemory are at least partially independent of the severity of psychosis symptoms and outlast acute delusions or hallucinations. It is possible that acute stages of psychosis, in contrast, are rather reflected by a tendency to generally attribute overconfidence to memories, independent of their correctness (Moritz and Woodward, 2002). Kircher et al. (2007) assumed that these non-significant correlations hint towards a trait feature of self-reflectivity in schizophrenia. In this case, these metamemory biases might be a stable part of psychosis development and might also be found in patients with an at risk mental state of psychosis. It might also be considered that due to a highly mixed constellation of acute and non-acute patients in our study, symptom severity scores were rather low and low variances of scores might have led to reduced correlation coefficients. However, these symptom scores resemble those of studies with similar patient groups (e.g. Moritz et al., 2011).

Though we did not find any associations between antipsychotic or anxiolytic treatment and metamemory performance, it is still possible that there are medication effects influencing our results. Inclusion criteria regarding antipsychotic treatment strongly differ between studies. While previous results suggested that treatment with lorazepam has no influence on evaluation accuracies of people's judgements of learning (Izaute and Bacon, 2005), it has been hypothesized that second generation antipsychotic agents might reduce metacognitive biases by correcting the interplay between cognitive performance and reasoning abilities or by adjusting the liberal threshold of acceptance (Moritz and Woodward, 2004). This assumption, which cannot be supported by our results, should be investigated further in studies with drug-naïve patients. Such investigations could increase sensitivity and abolish medication effects to unmask relevant associations between positive symptomatology and metamemory biases.

As expected, group comparisons revealed not only deficits in the recognition of studied words in the patient group, but also marked 
deficits in neuropsychological functioning in all tested domains of the MCCB (see also Kern et al. (2011)) and the WCST. We identified correlations in the total group between metamemory indices and two neuropsychological domains. Worse performance in executive functions (attention allocation and set shifting abilities as measured by the WCST) as well as in working memory was associated with a higher knowledge corruption index. A higher proportion of errors in the WCST was further associated with a lower confidence gap. Important to note, our results further provide evidence that memory and metamemory processes are partially different dimensions, as indicated by non-significant correlations between memory accuracy and memory monitoring. Speculatively, our results may indicate that impaired memory monitoring abilities and a tendency to attribute higher confidences to memory errors are modulated by these neurocognitive abilities. The mediating role of the prefrontal cortex for metacognitive capacities as well as executive functioning (Fernandez-Duque et al., 2000) gives one neuroanatomical explanation for this relation. Furthermore, the medial temporal lobe has been found to be involved in both, cognitive confidences (Chua et al., 2009) and working memory tasks (Olsen et al., 2009). These findings are in line with prior results (Mäntylä et al., 2010, Souchay et al., 2004). In their report, Mäntylä et al. discussed relations between executive functioning and subjective memory performance measured by questionnaires. Souchay et al. reported that prospective memory evaluations (feeling-of-knowing) were associated with executive functioning, measured by the WCST and a verbal fluency task. Our data does not support the prior assumption of metacognition being a rather autonomous and superordinate system (Kircher et al., 2007). Differences in neuropsychological tests probably contribute to deviating results. Whereas Kircher et al. (2007) used a digit-span task to measure working memory, in the MCCB this domain is represented by two tasks, namely letter-number-sequencing and block-tapping. Moreover, heterogeneity of metamemory tasks, especially regarding time-differences between learning and recognition phases, can affect working memory loads. In contrast to the knowledge corruption index, the confidence gap was not associated with working memory. These measures have slightly different targets and in contrast to the knowledge corruption index, the confidence gap as a measure of all confidence ratings presumably dilutes the component of memory accuracy more. It might therefore mirror more readily prefrontal activities. Furthermore, correlations regarding the confidence gap might have been masked by small variances.

This study is limited by its cross-sectional design excluding any assumptions about causality and by its explorative nature leading to the use of multiple correlations. Bonferroni corrections were implemented per research question. Further limitations concern the inclusion of inpatients during antipsychotic and anxiolytic treatment and with a chronic course of illness. We did not detect any correlations between medical treatment and performance but influences cannot be totally controlled by our statistical methods. Also, studies with patients in outpatient settings are needed to increase generalizability. With regard to the metamemory task, we used a rather narrow Likert-scale to evaluate confidence level ratings. Our scaling thus allowed less contrast between highconfident- and other ratings. It is known that the construction of the scale can influence self-awareness (Szczepanowski et al., 2013). This might have led to deviating evaluations of the knowledge corruption index.

In conclusion, this study replicated prior findings of a reduced confidence gap in schizophrenia. Importantly, this metamemory bias appears to be at least partially independent of current symptoms and might persist after and before acute delusions. Furthermore, correlations between knowledge corruption and executive functions as well as working memory, and between the confidence gap and executive functioning were accentuated by the data, giving insight into mechanisms of metamemory biases.
We emphasize to properly consider individual differences in these neurocognitive abilities in examinations of metacognition. Similar to the jumping to conclusion bias, which has been shown to be predictive for vocational abilities in schizophrenia (Andreou et al., 2014), the exploration of biases in memory evaluation can give us important insight into functional outcomes of schizophrenia. The results suggest that deviations in cognitive confidence are stable parameters within the psychosis spectrum. They might be associated with an increased risk of psychosis development. Knowledge about mechanisms of cognitive biases and processes in the development of psychosis symptoms will improve early treatment options, as the meta-cognitive training (Moritz et al., 2011). In perspective, investigations of drug-naïve patients with a first episode of psychosis and patients at risk for psychosis will improve our knowledge about associations between neurocognition and metamemory in the transition to psychosis.

\section{Funding}

S. Eifler was supported by a Grant of Heidelberg University(LGFG 2012-117). F.S. was supported by Grants of the Evangelisches Studienwerk and of Deutscher Akademischer Austauschdienst (DAAD). D. M. received fundings by the Olympia-Morata Program. M.Z., A.M.-L., and P.K. were funded by the Deutsche Forschungsgesellschaft (DFG, http://www.dfg.de, projects ZI1253/3-1, ZI1253/3-2, KI 576/14-2, ME 1591/6-2).

The funders had no role in study design, data collection and analysis, decision to publish or preparation of the manuscript.

\section{Conflicts of interest}

S.E., F.R., F.S., R.V., D.M., C.E., and P.K. declare no conflicts of interest regarding this manuscript.

S. Englisch has received travel expenses and consultant fees from AstraZeneca, Bristol-Myers Squibb GmbH \& CoKGaA, Eli-Lilly, Janssen Cilag, Lundbeck, Otsuka Pharma and Pfizer Pharma and Servier. A.M.-L. receives consultant fees and travel expenses from AstraZeneca, Hoffmann-La Roche, Lundbeck Foundation. Speaker's fees from Pfizer Pharma, Lilly Deutschland, Glaxo SmithKline, Janssen Cilag, Bristol-Myers Squibb, Lundbeck and AstraZeneca. M. Z. receives unrestricted scientific grants of the European Research Advisory Board (ERAB), German Research Foundation (DFG), Pfizer Pharma GmbH, Bristol Myers Squibb Pharmaceuticals and Servier; further speaker and travel grants were provided from Astra Zeneca, Lilly, Pfizer Pharma GmbH, Bristol Myers Squibb Pharmaceuticals, Otsuka, Servier, Lundbeck, Janssen Cilag, Roche and Trommsdorff.

\section{Acknowledgments}

We thank staff and patients of the Department for Psychiatry and Psychotherapy, in particular inpatient unit 5a, for their support and participation. 


\section{References}

Addington, D., Addington, J., Maticka-Tyndale, E., 1993. Assessing depression in schizophrenia: the calgary depression scale. British Journal of Psychiatry 163 39-44.

Aleman, A., Hijman, R., de Haan, E.H.F., Kahn, R.S., 1999. Memory impairment in schizophrenia: a meta-analysis. The American Journal of Psychiatry 156 (9), 1358-1366.

Andreasen, N.C., 1989. The scale for the assessment of negative symptoms (SANS): conceptual and theoretical foundations. British Journal of Psychiatry - Supplement 7, 49-58.

Andreasen, N.C., Carpenter, W.T., Kane, J.M., Lasser, R., Marder, S., Weinberger, D., 2005. Remission in schizophrenia: proposed criteria and rationale for consensus. American Journal of Psychiatry 162 (3), 441-449.

Andreasen, N.C., Pressler, M., Nopoulos, P., Miller, D., Ho, B.C., 2010. Antipsychotic dose equivalents and dose-years: a standardized method for comparing exposure to different drugs. Biological Psychiatry 67, 255-262.

Andreou, C., Moritz, S., Veith, K., Veckenstedt, R., Naber, D., 2013. Dopaminergic modulation of probabilistic reasoning and overconfidence in errors: a doubleblind study. Schizophrenia Bulletin 40 (3), 558-565.

Andreou, C., Treszl, A., Roesch-Ely, D., Köther, U., Veckenstedt, R., Moritz, S., 2014 Investigation of the role of the jumping-to-conclusions bias for short-term functional outcome in schizophrenia. Psychiatry Research 218 (3), 341-347. http://dx.doi.org/10.1016/j.psychres.2014.04.040.

Assaf, M., Rivkin, P., Kraut, M.A., Calhoun, V., Hart, J.r., Pearlson, G., 2007. Schizophrenia and semantic memory. In: Hart, J.r. Kraut, M.A. (Eds.), Neural Basis Of Semantic Memory. Cambridge University Press, New York, NY, pp. 133-145. http://dx.doi.org/10.1017/CBO9780511544965.006.

Bacon, E., Danion, J.M., Kauffmann-Muller, F., Bruant, A., 2001. Consciousness in schizophrenia: a metacognitive approach to semantic memory. Consciousness and Cognition 10 (4), 473-484.

Bacon, E., Huet, N., Danion, J.M., 2011. Metamemory knowledge and beliefs in patients with schizophrenia and how these relate to objective cognitive abilities. Consciousness and Cognition 20 (4), 1315-1326.

Bacon, E., Izaute, M., Danion, J.M., 2007. Preserved memory monitoring but impaired memory control during episodic encoding in patients with schizophrenia. Journal of the International Neuropsychological Society 13 (2) 219-227.

Benedict, R., 1997. Brief Visuospatial Memory Test-Revised: Professional Manual. Psychological Assessment Resources Inc., Odessa, FL.

Benedict, R., Schretlen, D., Groninger, L., Brandt, J., 1998. Hopkins verbal learning test-revised: normative data and analysis of inter-form and test-retest reliability. The Clinical Neuropsychologist 12 (1), 43-55.

Chua, E.F., Schacter, D.L., Sperling, R.A., 2009. Neural correlates of metamemory: comparison of feeling-of-knowing and retrospective confidence judgments. Journal of Cognitive Neuroscience 21, 7151-7165.

Corlett, P.R., Simons, J.S., Pigott, J.S., Gardner, J.M., Murray, G.K., Krystal, J.H. Fletcher, P.C., 2009. Illusions and delusions: relating experimentally-induced false memories to anomalous experiences and ideas. Frontiers in Behavioral Neuroscience 3 (53), 1-9.

Danion, J.M., Gokalsing, E., Robert, P., Massin-Krauss, M., Bacon, E., 2001. Defective relationship between subjective experience and behavior in schizophrenia. The American Journal of Psychiatry 158 (12), 2064-2066.

Deese, J., 1959. On the prediction of occurrence of particular verbal intrusions in immediate recall. Journal of Experimental Psychology 58 (1), 17-22.

Drake, R., Haddock, G., Tarrier, N., Bentall, R., Lewis, S., 2007. The Psychotic Symptom Rating Scales (PSYRATS): their usefulness and properties in first episode psychosis. Schizophrenia Research 89 (1-3), 119-122.

Drühe-Wienholt, C.M., Wienholt, W., 1998. Computergestütztes Kartensortierverfahren [Computer software]. Swets Test Services, Frankfurt, Germany.

Eifler, S., Rausch, F., Schirmbeck, F., Veckenstedt, R., Englisch, S., Meyer-Lindenberg, A., Kirsch., P., Zink, M., 2014. Neurocognitive capabilities modulate the integration of evidence in schizophrenia. Psychiatry Research 219 (1), 72-78,

Esslinger, C., Braun, U., Schirmbeck, F., Santos, A., Meyer-Lindenberg, A., Zink, M. Kirsch, P., 2013. Activation of midbrain and ventral striatal regions implicate salience processing during a modified beads task. PLoS One 8 (3), e58536.

Faul, F., Erdfelder, E., Lang, A.-G., Buchner, A., 2007. G*Power 3: a flexible statistical power analysis program for the social, behavioral, and biomedical sciences. Behavior Research Methods 39, 175-191.

Fernandez-Duque, D., Baird, J.A., Posner, M.I., 2000. Executive attention and metacognitive regulation. Consciousness and Cognition 9 (2), 288-307.

Fine, C., Gardner, M., Craigie, J., Gold, I., 2007. Hopping, skipping or jumping to conclusions? Clarifying the role of the JTC bias in delusions. Cognitive Neuropsychiatry $12(1), 46-77$.

Fioravanti, M., Carlone, O., Vitale, B., Cinti, M.E., Clare, L., 2005. A meta-analysis of cognitive deficits in adults with a diagnosis of schizophrenia. Neuropsychology Review 15, 73-95.

Flavell, J.H., 1971. First discussant's comments: what is memory development the development of? Human Development 14 (4), 272-278.

Fletcher, P.C. Frith, C.D., 2009. Perceiving is believing: a Bayesian approach to explaining the positive symptoms of schizophrenia. Nature Reviews Neuroscience $10(1), 48-58$.

Fusar-Poli, P., Meyer-Lindenberg, A., 2012a. Striatal presynaptic dopamine in schizophrenia, part $\mathrm{i}$ : meta-analysis of dopamine active transporter (DAT) density. Schizophrenia Bulletin 39 (1), 22-32.
Fusar-Poli, P., Meyer-Lindenberg, A., 2012b. Striatal presynaptic dopamine in schizophrenia, part II: meta-analysis of [18F/11C]-DOPA PET studies. Schizophrenia Bulletin 39 (1), 33-42.

Gaweda, L., Mikula, J., Szelenbaum, W., Kokoszka, A., 2014. Towards a cognitive model of hallucinations in the course of alcohol dependence? A source monitoring-based pilot study. Psychological Medicine 44, 2763-2773.

Gaweda, L., Moritz, S. Kokoszka, A, 2012. Impaired discrimination between imagined and performed actions in schizophrenia. Psychiatry Research 195, $1-8$.

Gaweda, L., Woodward, T.S., Moritz, S., Kokoszka, A., 2013. Impaired action selfmonitoring in schizophrenia patients with auditory hallucinations. Schizophrenia Research 144, 72-79.

Gras-Vincendon, A., Danion, J.M., Grangé, D., Bilik, M., 1994. Explicit memory repetition, priming and cognitive skill learning in schizophrenia. Schizophrenia Research 13 (2), 117-126.

Haddock, G., McCarron, J., Tarrier, N., Faragher, E.B., 1999. Scales to measure dimensions of hallucinations and delusions: the psychotic symptom rating scales (PSYRATS). Psychological Medicine 29 (4), 879-889.

Heinrichs, R.W., Zakzanis, K.K., 1998. Neurocognitive deficit in schizophrenia: a quantitative review of the evidence. Neuropsychology 12 (3), 426-445.

Iverson, G.L., Brooks, B.L., White, T., Stern, R.A., 2008. Neuropsychological assessment battery: introduction and advanced interpretation. In: Horton, A.M. Wedding, D (Eds.), The Neuropsychology Handbook, 3rd ed. Springer Publishing Co, New York, NY, pp. 279-343.

Izaute, M., Bacon, E., 2005. Specific effects of an amnesic drug: effect of lorazepam on study time allocation and on judgment of learning. Neuropsychopharmacology 30 (1), 196-204.

Jones, S.H., Thornicroft, G., Coffey, M., Dunn, G., 1995. A brief mental health outcome scale: reliability and validity of the Global Assessment of Functioning (GAF). British Journal of Psychiatry 166 (5), 654-659.

Jolley, S, Thompson, C, Hurley, J, Medin, E, Butler, L, Bebbington, P, Dunn, G, Freeman, P., Fowler, D, Kuipers, E, Garety, P., 2014. Psychiatry Research 219 (2), 275-282. http://dx.doi.org/10.1016/j.psychres.2014.05.051.

Kapur, S., 2003. Psychosis as a state of aberrant salience: a framework linking biology, phenomenology, and pharmacology in schizophrenia. The American Journal of Psychiatry 160 (1), 13-23.

Kay, S.R., Fiszbein, A., Opler, L.A., 1987. The positive and negative syndrome scale (PANSS) for schizophrenia. Schizophrenia Bulletin 13 (2), 261-276.

Keefe, R.S.E., Harvey, P.D., Goldberg, T.E., Gold, J.M., Walker, T.M., Kennel, C., Hawkins, K., 2008. Norms and standardization of the Brief Assessment of Cognition in Schizophrenia (BACS). Schizophrenia Research 102 (1-3), 108-115.

Kern, R.S., Gold, J.M., Dickinson, D., Green, M.F., Nuechterlein, K.H., Baade, L.E., Keefe, R. S.E, Mesholam-Gately, R.I., Seidman, L.J., Lee, C., Sugar, C.A., Marder, S.R., 2011. The MCCB impairment profile for schizophrenia outpatients: results from the MATRICS psychometric and standardization study. Schizophrenia Research 126 (1-3), 124-131.

Kircher, T.T.J., Koch, K., Stottmeister, F., Durst, V., 2007. Metacognition and reflexivity in patients with schizophrenia. Psychopathology 40 (4), 254-260.

Knye, N., Roth, M., Westhus, W., Heine, A., 2004. Continous Performance Test. Hogrefe, Göttingen, Germany.

Köther, U., Veckenstedt, R., Vitzthum, F., Roesch-Ely, D., Pfueller, U., Scheu, F., Moritz, S., 2012. "Don't give me that look" - overconfidence in false mental state perception in schizophrenia. Psychiatry Research 196 (1), 1-8.

Leavitt, V.M., Goldberg, T.E., 2009. Episodic memory in schizophrenia. Neuropsychology Review 19, 312-323.

Lee, J., Park, S., 2005. Working memory impairments in schizophrenia: a metaanalysis. Journal of Abnormal Psychology 114, 599-611.

Lehrl, S., 2005. Mehrfachwahl-Wortschatz-Intelligenztest MWT-B, unveränderte Auflage ed.5. Spitta Verlag, Balingen, Germany.

Lysaker, P.H., Warman, D.M., Dimaggio, G., Procacci, M., LaRocco, V.A., Clark, L.K. Dike, C.A., Nicol'o, G., 2008. Metacognition in schizophrenia: associations with multiple assessments of executive function. Journal of Nervous and Mental Disease 196 (5), 384-389.

Mäntylä, T., Rönnlund, M., Kliegel, M., 2010. Components of executive functioning in metamemory. Applied Neuropsychology 17 (4), 289-298.

Moritz, S., Gläscher, J., Sommer, T., Buechel, C., Braus, D.F., 2006c. Neural correlates of memory confidence. Neuroimage 33 (4), 1188-1193.

Moritz, S., Veckenstedt, R., Randjbar, S., Vitzthum, F., Woodward, T.S., 2011 Antipsychotic treatment beyond antipsych motics: metacognitive intervention for schizophrenia patients improves delusional symptoms. Psychological Medicine 41 (9), 1823-1832.

Moritz, S., Woodward, T.S., 2002. Memory confidence and false memories in schizophrenia. Journal of Nervous and Mental Disease 190 (9), 641-643.

Moritz, S., Woodward, T.S., 2004. Plausibility judgement in schizophrenic patients: evidence for a liberal acceptance bias. German Journal of Psychiatry 7, 66-74.

Moritz, S., Woodward, T.S., 2006a. Metacognitive control over false memories: a key determinant of delusional thinking. Current Psychiatric Report 8, 184-190.

Moritz, S., Woodward, T.S., 2006b. The contribution of metamemory deficits to schizophrenia. Journal of Abnormal Psychology 115 (1), 15-25.

Moritz, S., Woodward, T.S., Chen, E., 2006d. Investigation of metamemory dysfunctions in first-episode schizophrenia. Schizophrenia Research 81, 247-252.

Moritz, S., Woodward, T.S., Cuttler, C., Whitman, J.C., Watson, J.M., 2004. False memories in schizophrenia. Neuropsychology 18 (2), 276-283.

Moritz, S., Woodward, T.S., Jelinek, L., Klinge, R., 2008. Memory and metamemory in schizophrenia: a liberal acceptance account of psychosis. Psychological Medicine 38 (6), 825-832. 
Moritz, S., Woodward, T.S., Rodriguez-Raecke, R., 2006e. Patients with schizophrenia do not produce more false memories than controls but are more confident in them. Psychological Medicine 36 (5), 659-667.

Moritz, S., Woodward, T.S., Ruff, C.C., 2003. Source monitoring and memory confidence in schizophrenia. Psychological Medicine 33 (1), 131-139.

Moritz, S., Woodward, T.S., Whitman, J.C., Cuttler, C., 2005. Confidence in errors as a possible basis for delusions in schizophrenia. Journal of Nervous and Mental Disease 193 (1), 9-16.

Moritz, S., Woznica, A., Andreou, C., Köther, U., 2012. Response confidence for emotion perception in schizophrenia using a Continuous Facial Sequence Task. Psychiatry Research 200 (2-3), 202-207.

National Institute of Mental Health, 1970. Chapter 12- CGI. Clinical Global Impression. In: Guy, W. (Ed.), EDCEU Assessment Manual for Psychopharmacology. U.S. Department of Health, Education, and Welfare, Rockeville, MD, pp. 217-222.

Nelson, T.O., Narens, L., 1994. Why investigate metacognition?. In: Metcalfe, J, Shimamura, A.P. (Eds.), Metacognition: Knowing About Knowing. MIT Press, Cambridge, MA, pp. 1-25.

Nuechterlein, K.H., Green, M.F., 2006. MCCB MATRICS Consensus Cognitive Battery [Computer software]. The Regents of the University of California, Los Angeles.

Nuechterlein, K.H., Green, M.F., Kern, R.S., Baade, L.E., Barch, D.M., Cohen, J.D., Essock, S., Fenton, W.S., Frese, F.J., Gold, J.M., Goldberg, T., Heaton, R.K., Keefe, R. S., Kraemer, H., Mesholam-Gately, R., Seidman, L.J., Stover, E., Weinberger, D.R., Young, A.S., Zalcman, S., Marder, S.R., 2008. The MATRICS consensus cognitive battery, part 1: test selection, reliability, and validity. American Journal of Psychiatry 165 (2), 203-213.

Olsen, R.K., Nichols, E.A., Chen, J., Hunt, J.F., Glover, G.H., Gabrieli, J.D., Wagner, A.D. 2009. Performance-related sustained and anticipatory activity in human medial temporal lobe during delayed match-to-sample. Journal of Neuroscience 29, $11880-11890$.

Patrick, D.L., Burns, T., Morosini, P., Rothman, M., Gagnon, D.D., Wild, D., Adriaenssen, I., 2009. Reliability, validity and ability to detect change of the clinicianrated personal and social performance scale in patients with acute symptoms of schizophrenia. Current Medical Research and Opinion 25 (2), 325-338.

Peters, M.J.V., Hauschildt, M., Moritz, S., Jelinek, L., 2013. Impact of emotionality on memory and meta-memory in schizophrenia using video sequences. Journal of Behavior Therapy and Experimental Psychiatry 44 (1), 77-83.
Rausch, F., Mier, D., Eifler, S., Esslinger, C., Schilling, C., Schirmbeck, F., Englisch, D., Meyer-Lindenberg, A., Kirsch, P., Zink, M., 2014. Reduced activation in ventral striatum and ventral tegmental area during probabilistic decision-making in schizophrenia. Schizophrenia Research 156, 143-149.

Reitan, R.M., Wolfson, D., 1995. Category test and trail making test as measures of frontal lobe functions. Clinical Neuropsychologist 9 (1), 50-56.

Roediger III, H.L., McDermott, K.B., 1995. Creating false memories: remembering words not presented in lists. Journal of Experimental Psychology: Learning, Memory, and Cognition 21 (4), 803-814.

Sacher, M., Taconnat, L., Souchay, C., Isingrini, M., 2009. Divided attention at encoding: effect on feeling-of-knowing. Consciousness and Cognition 18 (3), 754-761.

Saß, H., Wittchen, H., Zaudig, M., 2000. Diagnostisches und statistisches Manual psychischer Störungen DSM-IV. Hogrefe, Göttingen, Germany.

Schneider, S.D., Jelinek, L., Lincoln, T.M., Moritz, S., 2011. What happened to the voices? A fine-grained analysis of how hallucinations and delusions change under psychiatric treatment. Psychiatry Research 188 (1), 13-17.

Sheehan, D.V., Lecrubier, Y., Sheehan, K.H., Amorim, P., Janavs, J., Weiller, E., Hergueta, T., Baker, R., Dunbar, G.C., 1998. The Mini-International Neuropsychiatric Interview (M.I.N.I): the development and validation of a structured diagnostic psychiatric interview for DSM-IV and ICD-10. Journal of Clinical Psychiatry 59 (20), 22-33.

Shimamura, A.P., 2000. Toward a cognitive neuroscience of metacognition. Consciousness and Cognition 9 (2), 313-323.

Souchay, C., Isingrini, M., Clarys, D., Taconnat, L., 2004. Executive functioning and judgment-of-learning versus feeling-of-knowing in older adults. Experimental Aging Research 30, 47-62.

Speechley, W.J., Whitman, J.C., Woodward, T.S., 2010. The contribution of hypersalience to the 'jumping to conclusions' bias associated with delusions in schizophrenia. Journal of Psychiatry and Neuroscience 35 (1), 7-17.

Szczepanowski, R., Traczyk, J., Wierzchon, M., Cleeremans, A., 2013. The perception of visual emotion: comparing different measures of awareness. Consciousness and Cognition: An International Journal 22, 212-220.

Wechsler, D., 1997. Wechsler Memory Scale (Administrations and Scoring Manual), Third ed. Psychological Corporation, San Antonio, Texas.

Woodward, T.S., Menon, M., Hu, X., Keefe, R.S., 2006. Optimization of a multinomial model for investigating hallucinations and delusions with source monitoring. Schizophrenia Research 85, 106-112. 